\title{
Incidental Lingual Thyroid Informs Surgery
}

\author{
Alyce Goode ${ }^{1}$, Cheryl McKellar ${ }^{1}$, Melanie Carter $^{1}$, Laura Skelly ${ }^{1}$, Lacey Greene ${ }^{1}$, George Roy ${ }^{2}$, \\ Matthew Haase ${ }^{2}$, and Geoffrey M. Currie ${ }^{1,3}$ \\ ${ }^{I}$ Faculty of Science, Charles Sturt University, Wagga Wagga, Australia; ${ }^{2}$ Regional Imaging, IMed Network, Wagga Wagga, Australia; \\ and ${ }^{3}$ Australian School of Advanced Medicine, Macquarie University, Sydney, Australia
}

This case study presents an incidental noting of a lingual thyroid on thyroid scintigraphy that had implications in later breast surgery. This information changed patient management and mitigated risk during intubation for breast cancer surgery.

Key Words: thyroid scan; lingual thyroid; intubation; incidental; endocrine; SPECT/CT

J Nucl Med Technol 2015; 43:66-67

DOI: 10.2967/jnmt.114.142075

\section{A}

difficult intubation during surgery can have fatal consequences (1). The uncommon embryologic condition of an ectopic lingual thyroid can pose difficulties with intubation because of hemorrhaging and airway compression (2), especially when the presence of a lingual thyroid is unknown before intubation. This case study presents a patient in whom incidental detection of a lingual thyroid during thyroid scintigraphy provided valuable information to inform later breast cancer surgery.

\section{CASE HISTORY}

A 67-y-old woman had a past history of throat discomfort suspected to be a lingual thyroid. On a recent presentation, she reported heavy breathing, a "husky feeling," and persisting throat discomfort. It was noted that the patient was taking thyroxin and had a euthyroid status. Nasoendoscopy revealed a mass in the base of the tongue and a normal larynx. The patient was referred for a nuclear medicine thyroid study. The thyroid scanning was performed $3 \mathrm{wk}$ after thyroxin cessation. Approximately $180 \mathrm{MBq}$ of ${ }^{99 \mathrm{~m}} \mathrm{Tc}$-pertechnetate were administered intravenously, with imaging commencing $15 \mathrm{~min}$ after administration. Standard planar anterior, anterior oblique, and marker images were obtained using low-energy, highresolution collimation, a matrix of $256 \times 256$, and a zoom of $\times 2.5$ set for a window peak of $140.5 \mathrm{keV} \pm 10 \%$. Low-dose SPECT/CT was subsequently performed, with a matrix of $128 \times 128$, a zoom of $\times 1.28$, and step-and-shoot mode at $8 \mathrm{~s} /$ frame for 120 frames for the SPECT component and $1.25-\mathrm{mm}$ slices at $120 \mathrm{kV}$ and $80 \mathrm{~mA}$ for the CT component.

Received Apr. 23, 2014; revision accepted Jun. 23, 2014.

For correspondence or reprints contact: Geoffrey M. Currie, Faculty of Science, Locked Bag 588, Charles Sturt University, Wagga Wagga 2678, Australia.

E-mail: gcurrie@csu.edu.au

Published online Sep. 4, 2014.

COPYRIGHT (C) 2015 by the Society of Nuclear Medicine and Molecular Imaging, Inc.
All imaging was performed on a Discovery SPECT/CT system (GE Healthcare).

The nuclear medicine thyroid study demonstrated a welldefined area of increased activity in the midline of the mouth consistent with a lingual thyroid (Fig. 1). The SPECT/CT images revealed that the area of uptake corresponded to an ill-defined, slightly hyperdense, soft-tissue lesion, located posterior to the tongue (Fig. 2). Areas of calcification could be seen within the lingual thyroid. Although the significance of this calcification was unclear, it may have been dystrophic and the possibility of an underlying malignancy could not be dismissed. No other thyroid tissue was seen in the suprasternal location of the thyroid bed. A 3-cm lingual thyroid was confirmed on specialist (otolaryngologist) physical examination. Given that the patient was symptomatic and there was calcification, elective excision was recommended. The lingual thyroid was not excised.

Three months after identification of the lingual thyroid, self-examination revealed a mass in the right breast. The $16 \times 14 \mathrm{~mm}$ mass was suggestive of malignancy on both mammography and ultrasound, and surgery was planned within the subsequent $10 \mathrm{~d}$ (wide local excision with sentinel node dissection). Incidental examination of the patients' broader medical history by the breast surgeon highlighted a potential difficulty during intubation. Prior knowledge of the lingual thyroid allowed contingency planning and avoidance of potential complications during anesthesia. Although no specific modifications to the procedure were undertaken, additional care and planning were used to circumvent potential complications.

\section{DISCUSSION}

An ectopic thyroid is a rare embryologic abnormality of the thyroid gland $(3,4)$. Ectopia occurs during descent of the thyroid from the foramen caecum to the normal position, as a result of maternal antibodies inhibiting thyroid antigens or persistent thyroglossal ductal epithelial tissue (3-5). An ectopic thyroid occurs in 1 in 100,000-300,000 people (2-4). A lingual thyroid, situated at the base of the tongue, is the most common location of ectopic thyroid tissue and is more common in females (2-4). A lingual thyroid can first present at any age, but the mean age at diagnosis is $40.5 \mathrm{y}$ (3). It is often asymptomatic, and the most common symptoms are due to growth of the lingual thyroid tissue $(3,4)$. This growth can occur as a result of metabolic stresses, including puberty, pregnancy, trauma, or menopause $(4,5)$. A lingual thyroid 

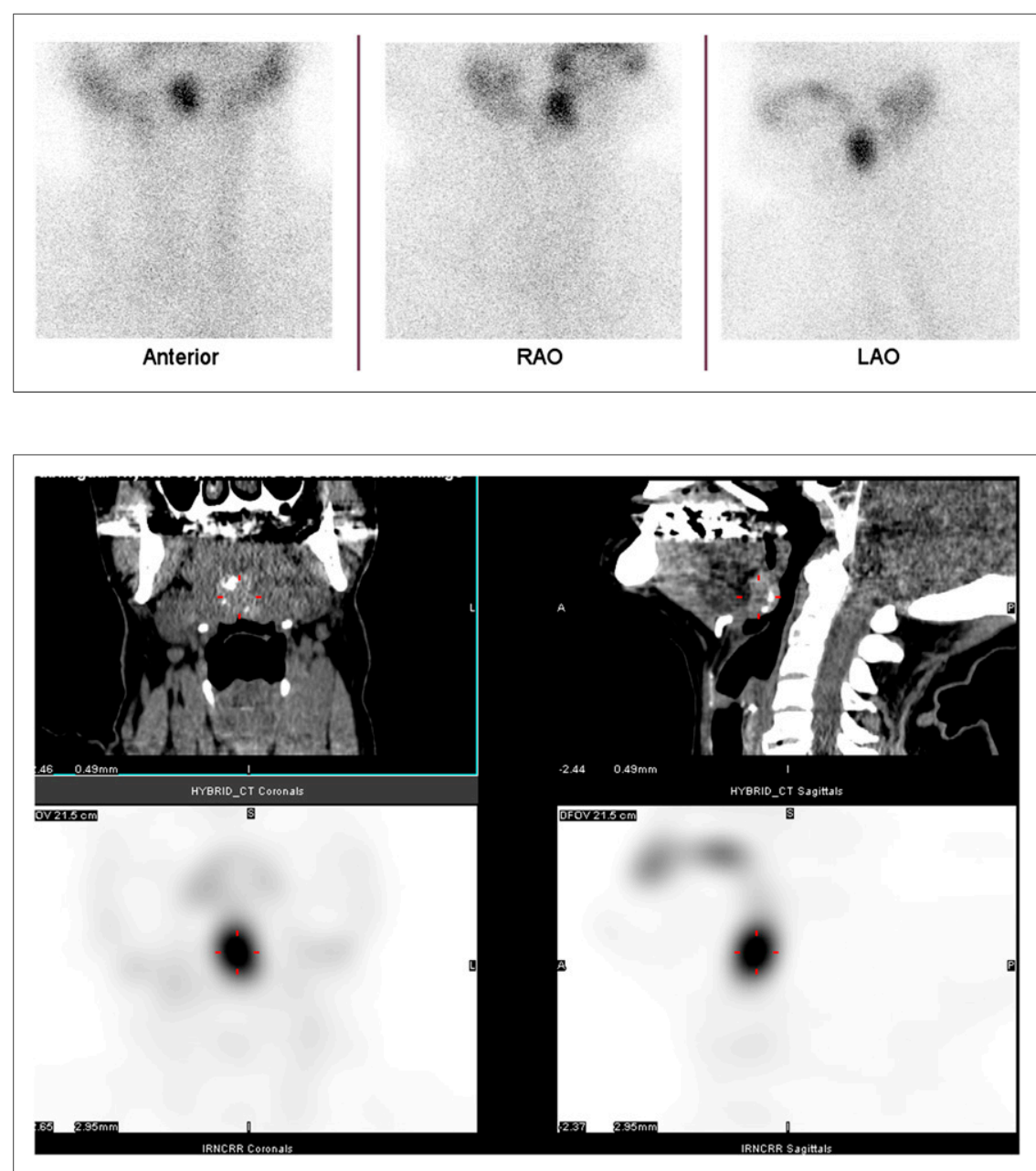

FIGURE 1. Planar anterior, right anterior oblique (RAO), and left anterior oblique (LAO) thyroid images raising suspicion of lingual thyroid.
FIGURE 2. SPECT/CT demonstrating midline localization of radionuclide in illdefined hyperdense soft-tissue lesion (cross hairs). can cause symptoms such as a cough, snoring, awareness of a foreign body, sleep apnea, dysphagia, dysphonia, and stomatolalia $(3,4)$. In severe cases, it can cause hemorrhage and respiratory obstruction $(3,4)$. Patients usually present with hypothyroidism, but some may present as euthyroid (3).

A difficult intubation can result in serious morbidity and mortality in clinical practice $(1,6)$. The Mallampati classification may be used to assess risk before intubation, with the highest risk being associated with an overly large base of tongue overshadowing the larynx $(1,6)$. This makes the larynx difficult to visualize during direct laryngoscopy (6). Because ectopic lingual thyroid tissue is usually at the base of tongue, it can result in a patient having an overly large base of tongue that can obscure the larynx $(2,4)$. Attempting intubation under such conditions may traumatize the glandular thyroid tissue, leading to hemorrhaging and possibly a compromised airway $(2,4)$.

In this case presentation, the SPECT/CT images demonstrated that the patient's lingual thyroid protruded into her pharynx. The patients' symptoms of throat discomfort, heavy breathing, and a husky feeling are consistent with this protuberance. Because of this compression, intubation for her breast cancer surgery may have been higher-risk had the information not been incidentally noted before surgery.

\section{CONCLUSION}

The hormonal changes that occur during menopause are likely to have caused the growth change in this patient's lingual thyroid, leading to its detection. Incidental noting of the presence of a lingual thyroid by the breast surgeon allowed contingency planning and risk mitigation during intubation. The case highlights the importance of presurgical evaluation of the broader medical history and the role of multidisciplinary communication.

\section{DISCLOSURE}

No potential conflict of interest relevant to this article was reported.

\section{REFERENCES}

1. Seo S, Lee J, Yu S, et al. Predictors of difficult intubation defined by the intubation difficulty scale (IDS): predictive value of 7 airway assessment factors. Korean J Anesthesiol. 2012;63:491-497.

2. Buckland RW, Pedley J. Lingual thyroid: a threat to the airway. Anaesthesia. 2000;55:1103-1105.

3. Noussios G, Anagnostis P, Goulis DG, Lappas D, Natsis K. Ectopic thyroid tissue: anatomical, clinical, and surgical implications of a rare entity. Eur J Endocrinol. 2011;165:375-382.

4. Toso A, Colombani F, Averono G, Aluffi P, Pia F. Lingual thyroid causing dysphagia and dyspnoea. Acta Otorhinolaryngol Ital. 2009;29:213-217.

5. Reddy P, Reddy K, Reddy R, Babu L. Ectopic lingual thyroid. Indian J Oral Sci. 2013;4:42-43.

6. Mallampati SR, Gatt SP, Gugino LD, et al. A clinical sign to predict difficult tracheal intubation: a prospective study. Can Anaesth Soc J. 1985;32:429-434. 